\title{
High-dose-rate brachytherapy as monotherapy in prostate cancer
}

Use of high-dose-rate brachytherapy as monotherapy for the treatment of intermediate-risk and high-risk prostate cancer is safe and effective, according to researchers in Japan.

Low-dose-rate permanent seed brachytherapy and temporary high-doserate brachytherapy are widely used for the treatment of early and locally advanced prostate cancer. High-dose-rate brachytherapy has historically been used in combination with external beam radiation therapy (EBRT), but more recently, interest has grown in the use of high-dose-rate brachytherapy as a monotherapy.

Which particular patients are suitable for use of high-dose-rate brachytherapy as a monotherapy, however, is a subject of debate. Some groups have argued that use of this therapy as a monotherapy is only suitable for lower risk patients, and that combination therapy with EBRT should be used for intermediate-risk and high-risk patients. "Other groups, however, including ours, have maintained there is no good reason for the addition of EBRT, even for the high-risk patients, because HDR-BT [high-doserate brachytherapy] was shown to provide adequate irradiation even for extracapsular lesions," say Yoshioka and colleagues, the authors of the latest paper. These researchers investigated use of high-dose-rate brachytherapy as a monotherapy in intermediate-risk and high-risk patients, with promising preliminary and interim results. Their most recent publication presents updated results from their study, with a median of 8 years of follow-up data.

Yoshioka and co-workers' final study population included 190 patients with intermediate-risk $(n=79)$ and high-risk $(n=111)$ localized prostate cancer treated with high-dose-rate brachytherapy as a monotherapy between 1995 and 2012. At 5 years, overall survival was 100\% in intermediate-risk patients and $93 \%$ in high-risk patients. At 8 years, overall survival was $96 \%$ in intermediate-risk patients and $81 \%$ in high-risk patients. Metastasis-free survival was $91 \%$ in intermediate-risk patients and $74 \%$ in high-risk patients at 8 years. Biochemical control rates were $91 \%$ in intermediate-risk patients and $77 \%$ in high-risk patients at 8 years.

The researchers report no incidences of grade 4 or 5 acute or late toxic effects. The cumulative incidence of late grade 3 genitourinary toxicity was $0 \%$ at 5 years and $1 \%$ at 8 years.

The cumulative incidence of late grade 3 gastrointestinal toxicity was $0 \%$ at 5 years and $2 \%$ at 8 years.

The authors state that their biochemical control rates and low rates of grade 3 late genitourinary and gastrointestinal toxicity rates are comparable to or better than those reported with other modalities of radiation therapy, and that high-doserate brachytherapy should be investigated further as a monotherapy in this patient group. 